\title{
Tecnura
}

\section{Salud ambiental del río Ranchería a través de macroinvertebrados acuáticos en el área de influencia del complejo carbonífero El Cerrejón}

\author{
Environmental health of the Ranchería river \\ through aquatic macroinvertebrates in the area of influence \\ of the El Cerrejón carboniferous complex
}

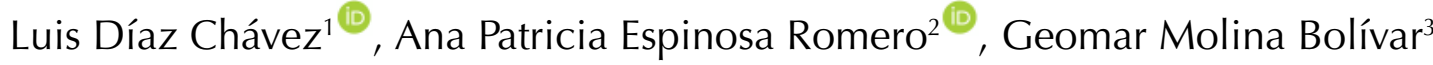

Fecha de recepción: 23 de Diciembre de 2019

Fecha de aceptación: 6 de Mayo de 2020

Cómo citar: Díaz-Chávez., L. Espinosa - Romero., A.P. y Molina-Bolívar., G. (2020). Salud ambiental del río Ranchería a través de macroinvertebrados acuáticos en el área de influencia del complejo carbonífero El Cerrejón. Tecnura, 24(65) 49-63. DOI: 10.14483/22487638.15773

\section{Resumen}

Objetivo: Esta investigación tuvo como objetivo evaluar la salud ambiental del ecosistema lótico río Ranchería en el área de influencia del complejo carbonífero El Cerrejón, a través de bioindicación con macroinvertebrados acuáticos.

Metodología: Se establecieron tres estaciones de monitoreo $\left(E_{1}, E_{2}\right.$ y $\left.E_{3}\right)$, en las cuales se realizaron mediciones de variables fisicoquímicas como: temperatura $\left({ }^{\circ} \mathrm{C}\right)$, oxígeno disuelto $(\mathrm{mg} / \mathrm{l}), \mathrm{pH}$, salinidad $(\% \mathrm{O})$, conductividad $(\mu \mathrm{S} / \mathrm{cm})$, turbidez (UNT), sólidos suspendidos totales $(\mathrm{mg} / \mathrm{l})$, cloruros $(\mathrm{mg} / \mathrm{l})$, amo$\operatorname{nios}(\mu \mathrm{g} / \mathrm{l})$, nitritos $-(\mu \mathrm{g} / \mathrm{l})$, nitratos $(\mu \mathrm{g} / \mathrm{l})$, fosfatos $(\mu \mathrm{g} / \mathrm{l})$ y demanda bioquímica de oxígeno (mg/l). Los macroinvertebrados acuáticos se colectaron a través de muestreo multihábitat por arrastre con red surber, red D-net y recolección de organismos bentónicos, usando nucleadores PVC. La calidad de las aguas en cada estación de monitoreo se clasificó mediante el índice BMWP/col.
Resultados: Como resultados relevantes, se encontró que las familias de macroinvertebrados acuáticos con más abundancia en $E_{1}$ fueron Baetidae, Coenagrionidae, Philopomamidae, Leptohyphidae y Leptophlebiidae; en $\mathrm{E}_{2}$ Culicidae, Hydrophilidae, Coenagrionidae, Psephenidae e Hydrobiidae, y en $E_{3}$, Coenagrionidae, Hydrobiidae, Belastomatidae $y$ Palaemonidae.

Conclusiones: Se concluye que $\mathrm{E}_{1}$ posee aguas de buena calidad $(\mathrm{BMWP} / \mathrm{col}=130 ;$ ASPT $=6,50)$, con alta diversidad y riqueza $\left(\mathrm{H}_{\mathrm{E} 1}^{\prime}=3,75\right.$ bits/ind; $\left.\mathrm{I}_{\mathrm{E} 1}=4,76\right) ; \mathrm{E}_{2}$ y $\mathrm{E}_{3}$ poseen aguas de calidad aceptable $(\mathrm{BMWP} / \mathrm{col}=65 ; \mathrm{ASPT}=5,00)$ y calidad dudosa $(\mathrm{BMWP} / \mathrm{col}=50 ; \mathrm{ASPT}=5,25)$, respectivamente, con mediana diversidad de familias $\left(\mathrm{H}_{\mathrm{E} 2}^{\prime}=2,97\right.$ bits/ ind $y \mathrm{H}_{\mathrm{E} 3}^{\prime}=2,32$ bits/ind) y mediana riqueza $\left(\mathrm{I}_{\mathrm{E} 2}=\right.$ 2,82 y $\left._{\mathrm{E} 3}=1,88\right)$.

Financiamiento: Universidad de La Guajira.

Palabras clave: Calidad de agua, bioindicadores, $\mathrm{BMWP} / \mathrm{col}$.

1 Ingeniero ambiental. Profesional de apoyo. Instituto de Estudios Ambientales y Aprovechamiento de Agua (INESAG), Universidad de La Guajira. Riohacha, Colombia. Contacto: luisdiazc@uniguajira.edu.co

2 Magistra en Desarrollo Rural, especialista en Gerencia Ambiental y Desarrollo Sostenible, ingeniera del Medio Ambiente. Directora del Programa de Ingeniería Ambiental y docente, Universidad de La Guajira. Riohacha, Colombia. Contacto: apespinosa@uniguajira.edu.co

3 Doctor en Ciencias Naturales para el Desarrollo, MSc en Ciencias Biológicas Mención Ecología Acuática, licenciado en Biología y Química. Docente de planta, Universidad de La Guajira. Riohacha, Colombia. Contacto: gmolina@uniguajira.edu.co 


\begin{abstract}
Objective: This research aimed to evaluate the environmental health of the lunatic ecosystem River Ranchería in the area of influence of the El Cerrejón coal complex, through bioindication with aquatic macroinvertebrates.

Methodology: Three monitoring stations were established $\left(E_{1}, E_{2}\right.$ y $\left.E_{3}\right)$, in which measurements of physicochemical variables were made such as: Temperature $\left({ }^{\circ} \mathrm{C}\right)$, Dissolved Oxigen $(\mathrm{mg} / \mathrm{L}), \mathrm{pH}$, Salinity $(\% \mathrm{O})$, Conductividad $(\mu \mathrm{S} / \mathrm{cm})$, Turbidity (UNT), Total Suspended Solids $(\mathrm{mg} / \mathrm{L})$, Chlorides $(\mathrm{mg} / \mathrm{L})$, Amonios $(\mu \mathrm{g} / \mathrm{L})$, Nitrites $(\mu \mathrm{g} / \mathrm{L})$, Nitrates $(\mu \mathrm{g} / \mathrm{L})$, Phosphates $(\mu \mathrm{g} / \mathrm{L})$ and Biochemical Oxygen Demand $(\mathrm{mg} / \mathrm{L})$. Aquatic macroinvertebrates were collected through multi-habitat sampling with surber net, D-net and PVC nucleators for collection of benthic organisms. Water quality at each monitoring station was classified using the BMWP/col index.
\end{abstract}

Results: As relevant results, it was found that the families of aquatic macroinvertebrates with the most abundance in $\mathrm{E}_{1}$ were Baetidae, Coenagrionidae, Philopomamidae, Leptohyphidae and Leptophlebiidae; in $\mathrm{E}_{2}$, Culicidae, Hydrophilidae, Coenagrionidae, Psephenidae e Hydrobiidae and in $E_{3^{\prime}}$ Coenagrionidae, Hydrobiidae, Belastomatidae and Palaemonidae.

Conclusions: It is concluded that $E_{1}$ has good quality waters (BMWP/col 130; ASPT 6.50), with high diversity and richness ( $\mathrm{H}^{\prime} \mathrm{E} 1 \times 3.75$ bits/ind; IE1 x 4.76); $E_{2}$ and $E_{3}$ have waters of acceptable quality (BMWP/ col x 65; ASPT 5.00) and dubious quality (BMWP/ col x 50; ASPT-5.25), respectively, with median diversity of families $\left(\mathrm{H}^{\prime} \mathrm{E} 2 \times 2.97\right.$ bits/ind and $\mathrm{H}^{\prime} \mathrm{E} 3 \times$ 2.32 bits/ind) and median wealth (IE2 x 2.82 and IE3 x 1.88).

Financing: Universidad de La Guajira.

Keywords: Water quality, bioindicators, BMWP/col.

\section{INTRODUCCIÓN}

Los ríos son ecosistemas dinámicos y complejos, con múltiples conexiones con otros ecosistemas, longitudinales (conexión río arriba-río abajo), laterales (conexión con la cuenca hidrográfica y vegetación de la ribera) y verticales (conexión con las aguas subterráneas y la precipitación) (Encalada, 2010). Estas particularidades les permiten brindar múltiples servicios ambientales que han sido utilizados como ejes para el desarrollo de la humanidad, con la consecuente contaminación y transformación de la morfología de sus cauces por la intervención antrópica asociada a grandes obras de infraestructura, afectando además, la calidad del agua con la disposición y vertimiento de residuos sólidos y líquidos, que tiene graves efectos en la biodiversidad acuática y en las funciones y servicios ecosistémicos que proveen.

Particularmente, el río Ranchería, considerado la principal fuente hídrica del departamento de La Guajira, alberga sobre su cuenca el desarrollo de muchas de las actividades económicas del departamento, incluyendo la minería de explotación de carbón a cielo abierto de El Cerrejón, y por su amplio recorrido a través de su superficie, baña con sus aguas a muchos de sus municipios que lo han convertido en el principal receptor de efluentes de los diferentes sistemas de tratamiento de aguas residuales, que no operan eficientemente en la remoción de la carga contaminante (Corpoguajira, 2011).

Diversos estudios han analizado la calidad del agua del río Ranchería (Molina-Bolívar y Jiménez-Pitre, 2017; Bolívar, Pitre y Correa, 2017; Argumedo, Sierra y Negrete, 2017; Pérez, Nardini y Galindo, 2018; Fuentes, Verbel, Villegas y Campos, 2019) y algunos de ellos (Pérez, Nardini y Galindo, 2018; Fuentes et al., 2019) han demostrado el deterioro de la calidad del agua a su paso por la mina de El Cerrejón, dados los vertimientos que realizan los centros poblados alrededor del cauce principal, el aumento de la turbiedad, la disminución de la velocidad del mismo y el excesivo 
transporte de material suspendido producto de los materiales de arrastre aguas arriba de la cuenca.

No obstante, la mayoría de estos estudios se han realizado desde el punto de vista fisicoquímico de las aguas del río Ranchería, y por ser reconocido ampliamente que estos solo reflejan las condiciones ambientales del ecosistema en el instante en que son evaluadas, se considera necesario incorporar procedimientos de bioindicación a través de macroinvertebrados acuáticos para evaluar la calidad de las aguas del río Ranchería, dado que los bioindicadores reflejan la dinámica ambiental del ecosistema en el tiempo y, por su simplicidad metodológica, rapidez en la obtención de los resultados y alta confiabilidad, se constituyen en una herramienta idónea para el análisis de calidad del agua en ecosistemas lóticos (Alba-Tercedor y Sánchez-Ortega, 1988; Medianero y Samaniego, 2004; López et al., 2005; Ortiz, 2005; Giacometti, 2019).

En la actualidad, son muchas las investigaciones de bioindicación a través de macroinvertebrados acuáticos que se han realizado en el mundo y en Colombia, puesto que estos son considerados como los mejores indicadores de calidad del agua (Correa, Machado y Roldán, 1981; Álvarez y Roldán, 1983; Arango y Roldán, 1983; Bedoya y Roldán, 1984; Roldán, 1985; Zúñiga de Cardoso, 1985; Alba-Tercedor y Sánchez-Ortega, 1988; Machado y Rincón, 1989; Pérez, 1999; Sánchez-Vélez y García-Núñez, 1999; Riss, Ospina y Gutiérrez, 2002; Vivas et al., 2002; Chalá et al., 2003; Guerrero-Bolaño, Manjarrés-Hernández y Núñez-Padilla, 2003; Roldán-Pérez, 2003, 2016; González y Lozano, 2004; Gutiérrez, Riss y Ospina, 2004; López et al., 2005; Molina, Fossati y Marín, 2006; Guinard, Ríos y Vega, 2013; Madera, Angulo, Díaz y Rojano, 2016; Murillo-Montoya, Mendoza-Mora, Restrepo-Bastidas y Rodríguez, 2018; Giacometti, 2019). Sin embargo, en el área de estudio, las investigaciones de la calidad de las aguas del río Ranchería a través de procesos de bioindicación son escasos. La literatura revisada solo reporta estudios de este tipo en la zona deltaica del río
Ranchería (Castro et al., 2005; Arteta-Bonivento, 2009; Castro et al., 2013).

En virtud de lo anterior, el objetivo de esta investigación fue evaluar la salud ambiental del río Ranchería a través macroinvertebrados acuáticos en el área de influencia del complejo carbonífero El Cerrejón, permitiendo conocer las condiciones de calidad de este ecosistema desde un enfoque biológico para una mejor administración del recurso por parte de las instituciones encargadas a esta labor.

\section{METODOLOGÍA}

\section{Área de estudio y estaciones de monitoreo}

El río Ranchería está localizado en el departamento de La Guajira (Colombia); nace en la Sierra Nevada de Santa Marta, en la laguna Chirigua a una altitud de 3875 msnm, y tiene una longitud aproximada de 248 km (Corpoguajira, 2011; Corpoguajira y Fundación Pro-Sierra Nevada de Santa Marta, 2012).

Para el desarrollo de este estudio se establecieron tres estaciones de monitoreo sobre el cauce principal del río Ranchería: $E_{1}, E_{2}$ y $E_{3}$ (figura 1), respectivamente georreferenciadas a través de un receptor GPS Garmin Etrex (tabla 1). La estación E1, tomada como blanco, se localizó aguas arriba del río Ranchería (cuenca alta) a la altura del corregimiento de Caracolí, Sabana de Manuela, municipio de San Juan del Cesar, se caracteriza porque el cauce presenta un ancho aproximado de $30 \mathrm{~m}$ con aguas turbulentas, cristalinas y profundidades promedios de $0,40 \mathrm{~m}$; su fondo se compone de abundante gravilla y rocas de gran tamaño, y en algunos sectores sus bordes presentan lodos finos con presencia de material vegetal de la flora circundante. Las estaciones $E_{2}$ y $E_{3}$ fueron situadas en el área de influencia del complejo carbonífero de El Cerrejón $\mathrm{E}_{1}$. $\mathrm{E}_{2}$ se localizó antes del complejo minero a $300 \mathrm{~m}$ de la población del corregimiento de Papayal, municipio de Barrancas, caracterizada por tener aguas torrentosas en época de Iluvias con 
coloración marrón oscuro atribuidas a la presencia de partículas en suspensión. En época de sequía sus aguas son quietas y transparentes con coloración verdosa producto de la presencia de microalgas del género Lemna; su profundidad promedio es de 0,60 m con fondos arenosos y lechos blandos en los bordes. La estación $\mathrm{E}_{3}$, ubicada aproximadamente entre 2-3 km del municipio de Albania, presenta una profundidad promedio de $030 \mathrm{~m}$ y un ancho aproximado de $10 \mathrm{~m}$, con aguas corrientes poco transparentes y lecho constituido por gravas y cantos rocosos afilados; en los extremos, el lecho presenta lodos finos.

\section{Medición de variables fisicoquímicas}

En las estaciones de monitoreo se realizaron mediciones in situ de las variables fisicoquímicas: temperatura (Temp.), oxígeno disuelto (OD), $\mathrm{pH}$, salinidad (Sal.), conductividad (Cond.), utilizando un multiparámetro WTW 3320 y turbidez (Turb.) a través de un turbidímetro HACH 2100P. Asimismo,

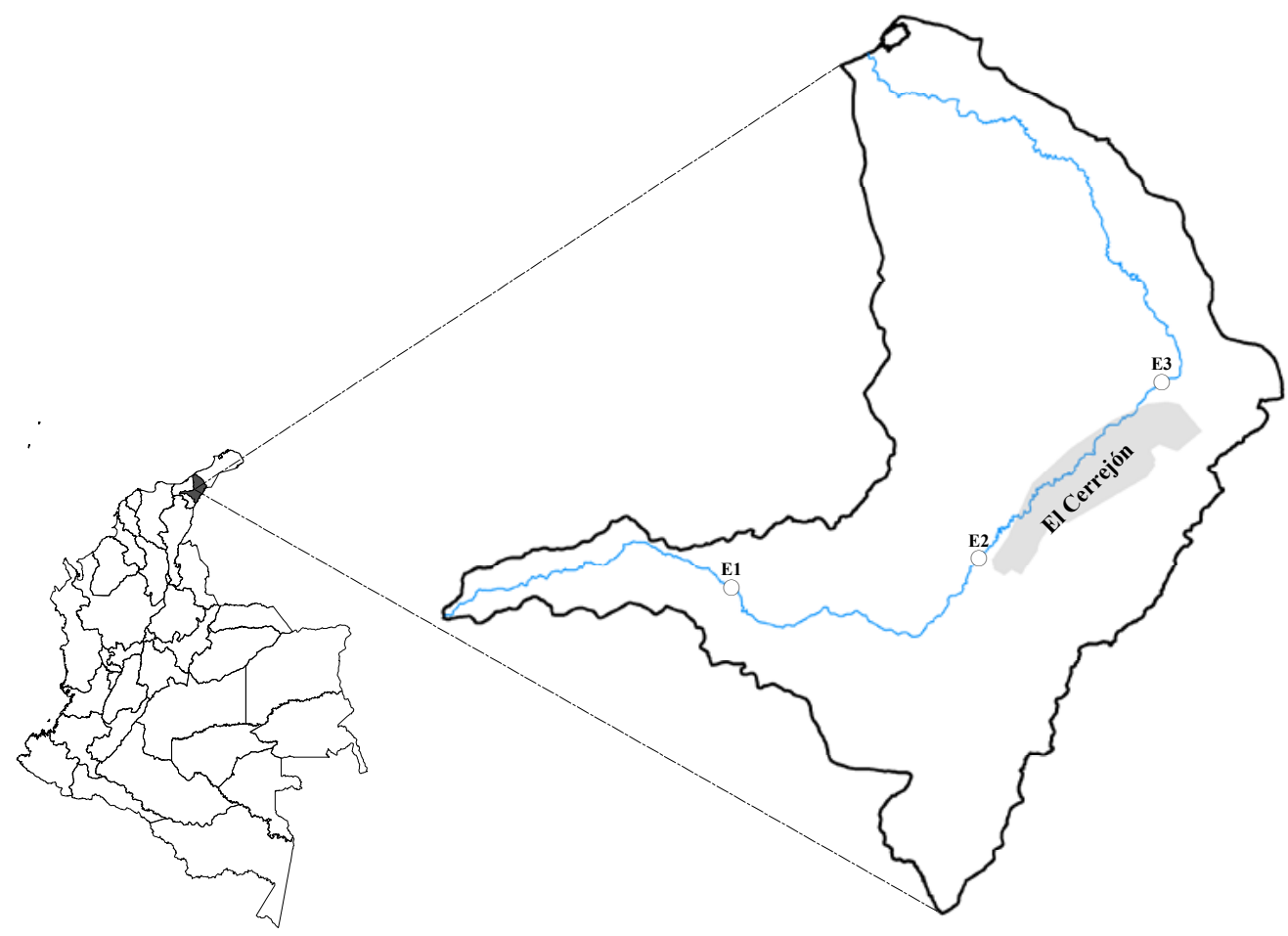

Figura 1. Localización del área de estudio

Fuente: elaboración propia.

Tabla 1. Geolocalización de las estaciones de monitoreo

\begin{tabular}{cccc}
\hline \multirow{2}{*}{ Estaciones de monitoreo } & \multicolumn{2}{c}{ Coordenadas } & \multirow{2}{*}{ Descripción de la zona } \\
\cline { 2 - 3 } & Norte & Este & \\
\hline$E_{1}$ & $10^{\circ} 57^{\prime} 12.85^{\prime \prime}$ & $73^{\circ} 03^{\prime} 31.66^{\prime \prime}$ & Corregimiento de Caracolí \\
\hline$E_{2}$ & $10^{\circ} 59^{\prime} 02.49^{\prime \prime}$ & $72^{\circ} 46^{\prime} 00.42^{\prime \prime}$ & Corregimiento de Papayal \\
\hline$E_{3}$ & $11^{\circ} 10^{\prime} 48.29^{\prime \prime}$ & $72^{\circ} 33^{\prime} 11.74^{\prime \prime}$ & Municipio de Albania \\
\hline
\end{tabular}

Fuente: elaboración propia. 
se tomaron muestras en botellas de polietileno de $500 \mathrm{ml}$ para la determinación de las concentraciones de sólidos suspendidos totales (SST), amonio $\left(\mathrm{NH}_{4}^{+}\right)$, nitritos $\left(\mathrm{NO}_{2}^{-}\right)$, nitratos $\left(\mathrm{NO}_{3}^{-}\right)$, fosfatos $\left(\mathrm{PO}_{4}^{-3}\right)$, cloruros $\left(\mathrm{Cl}^{-}\right)$y la demanda biológica de oxígeno $\left(\mathrm{DBO}_{5}\right)$, las cuales fueron trasladadas bajo refrigeración a $4{ }^{\circ} \mathrm{C}$ al laboratorio de calidad ambiental de la Universidad de La Guajira, para su análisis en un periodo de 8 horas después de la toma de muestras (APHA, et al., 2005). Estos monitoreos se realizaron abarcando un periodo de lluvias comprendido entre los meses de septiembre a octubre de 2014, y uno de sequía, de abril a junio de 2015, de acuerdo con el régimen bimodal de la zona de estudio.

\section{Recolección e identificación de macroinvertebrados acuáticos}

Los macroinvertebrados acuáticos se colectaron a través de un muestreo multihábitat, mediante una red Surber con luz de malla de $1 \mathrm{~mm}$, según la metodología recomendada por Roldán (1988) y Moreno (2008). Además, se utilizaron redes D-net para hacer barridos de los márgenes del río con vegetación y de este modo atrapar a los insectos nadadores o que viven adheridos a los tallos y hojas de la vegetación sumergida (Roldán, 1988); nucleadores de PVC de 2 " de diámetro para la colecta de organismos bentónicos y colecta manual de organismos adheridos a rocas y restos de vegetación (troncos y hojarasca). Los organismos encontrados fueron tomados con pinzas entomológicas y depositados en frascos de $30 \mathrm{ml}$, debidamente rotulados; las muestras se preservaron en alcohol al 70 \% (Posada et al., 2000), y posteriormente fueron trasladadas al Laboratorio de Ciencias Biológicas de la Universidad de La Guajira para su identificación a través del uso de claves taxonómicas (Roldán, 1988; Aquatic National, 2001; Fernández y Domínguez, 2001; Muñoz, 2004; Bouchard, Ferrington y Karius, 2004; Álvarez, 2005; McCafferty, 2014; Melo y Montemayor, 2014).

\section{Análisis de los datos}

El tratamiento de la información colectada consistió en la determinación de medidas de resumen para las variables fisicoquímicas entre estaciones de monitoreo, cálculo de la estructura numérica de los macroinvertebrados acuáticos a través de los índices de diversidad de Shannon-Weaver (Shannon, 1948) y riqueza de Margalef; clasificación de la calidad de las aguas en cada estación de monitoreo a través del índice BMWP/col (Roldán-Pérez, 2003), evaluación de las asociaciones entre las morfofamilias de macroinvertebrados encontradas y las variables fisicoquímicas medidas a través de un análisis de componentes principales usando el paquete estadístico R v.3.2.5.

\section{RESULTADOS}

Desde el punto de vista fisicoquímico, el resumen de las variables consideradas para el desarrollo de este estudio se expone en la tabla 2, donde es evidente el deterioro que presentan las aguas del río Ranchería, conforme su cauce principal hace su recorrido desde $E_{1}$ hasta $E_{3}$, presentándose un incremento significativo en la magnitud y variabilidad de los valores medios del contenido de sólidos en suspensión, las concentraciones de $\mathrm{Cl}$-, nutrientes $\left(\mathrm{NH}_{4}{ }^{+}, \mathrm{NO}_{2}{ }^{-}, \mathrm{NO}_{3}^{-}\right.$y $\left.\mathrm{PO}_{4}^{-3}\right)$ y la $\mathrm{DBO}_{5}$.

Considerando este punto de vista, una visión general del río Ranchería, en el área de estudio, muestra que el incremento de los valores de conductividad eléctrica, sumado a la disminución paulatina del oxígeno disuelto y el $\mathrm{pH}$, son indicativos de impactos en el ecosistema por el desarrollo de actividades antrópicas que, a causa de fluctuaciones en las concentraciones relativas de la totalidad de los iones, en la sumatoria de sus efectos a través del tiempo, muestran o evidencian cambios en la conductividad (Garbagnati, González, Antón y Mallea, 2005). Análogamente, el incremento en las concentraciones de nutrientes y $\mathrm{DBO}_{5}$ se traduce en deterioro de la calidad del agua por aportes de carga contaminante a través 
de efluentes vertidos sobre el cauce del río; sin embargo, el predominio de formas oxidadas del nitrógeno $\left(\mathrm{NO}_{2}^{-}\right.$y $\left.\mathrm{NO}_{3}^{-}\right)$, respecto a su forma reducida $\left(\mathrm{NH}_{4}^{+}\right)$, revela la capacidad de amortiguamiento del río sobre la contaminación y/o su capacidad de autodepuración, en tanto no se incrementen de forma drástica los aportes de contaminantes orgánicos (Castañé et al., 1998; Toro et al., 2002).

Por otro lado, también resalta la contaminación visual o estética que presentan las aguas del río Ranchería en su cuenca media $\left(E_{2}\right.$ y $\left.E_{3}\right)$, a causa de mayores valores de turbidez y sólidos suspendidos totales, atribuidos a la relación positiva y significativa que estas variables guardan con el incremento de precipitaciones sobre las cuencas; efecto que se agudiza en la medida que estas sufran de problemas de desforestación, sean de sustrato blando y fácilmente erosionables (Ortega et al., 1988), como ocurre en la cuenca del río Ranchería.

En general, la dinámica de las variables fisicoquímicas en este estudio corresponde con los valores reportados en otros estudios realizados en estaciones de monitoreo cercanas a las establecidas para el desarrollo de este, en todas las variables analizadas, exceptuando los valores obtenidos de nutrientes (grupo del nitrógeno y $\mathrm{PO}_{4}^{-3}$ ), que resultaron ser significativamente inferiores (Bonivento, 2010; Corpoguajira y Fundación Pro-Sierra Nevada de Santa Marta, 2012; Rangel, 2013).

Por otro lado, el muestreo multihábitat de macroinvertebrados acuáticos logró la colecta de 166 individuos representados por 3 filo, 5 clases, 11 órdenes y 30 familias. En general, los órdenes más representativos fueron Odonata (42 ind., $25,00 \%$ ) y Díptera (33 ind., 19,64\%), seguidos por Coleóptera (20 ind., 11,90 \%) y Hemíptera (16 ind., 9,52). Así mismo, las familias más representativas corresponden a Coenagrionidae (35 ind., $20, .83 \%$ ), Culicidae (25 ind., 14,88\%) y Baetidae (14 ind., 8,33\%), seguidas por Hydrobiidae (12 ind., 7,14 \%) y Hydrophilidae (11 ind., 6,55 \%).

La distribución espacial de los órdenes y morfofamilias encontradas en la zona de estudio se muestran en las figuras 2 y 3 , respectivamente, observándose mayor abundancia en $\mathrm{E}_{1}$ de los órdenes Ephemeroptera (24 ind.), Hemiptera (10 ind.), Odonata (10 ind.) y Tricoptera (7 ind.). En $E_{2}$ la mayor abundancia de individuos se obtuvo para los órdenes Díptera (29 ind.), Coleóptera (17 ind.) y Odonata (13 ind). Por último, $\mathrm{E}_{3}$ se encuentra mejor representada en cuanto a abundancia por los órdenes Odonata (19 ind.), Sorbeoconcha (7 ind.)

Tabla 2. Resumen de los valores de las variables fisicoquímicas expresadas como $\bar{x} \pm S$

\begin{tabular}{ccccc}
\hline \multirow{2}{*}{ Variable } & Unid. & $\mathbf{E}_{\mathbf{1}}$ & $\mathbf{E}_{\mathbf{2}}$ & $\mathbf{E}_{\mathbf{3}}$ \\
\cline { 3 - 5 } & & $\overline{\mathbf{x} \pm \mathbf{s}}$ & $\overline{\mathbf{x}} \pm \mathbf{s}$ & $\overline{\mathbf{x}} \mathbf{\text { s }}$ \\
\hline $\mathrm{Temp}$ & ${ }^{\circ} \mathrm{C}$ & $24,85 \pm 1,44$ & $31,45 \pm 2,72$ & $33,45 \pm 3,08$ \\
\hline $\mathrm{pH}$ & $\mathrm{Adim}$. & $8,2 \pm 0,44$ & $7,86 \pm 0,30$ & $7,83 \pm 0,20$ \\
\hline $\mathrm{OD}$ & $\mathrm{mg} / \mathrm{l}$ & $8,48 \pm 1,26$ & $6,80 \pm 2,14$ & $6,51 \pm 1,56$ \\
\hline $\mathrm{Sal}$ & $\%_{0}$ & $0,00 \pm 0,00$ & $0,00 \pm 0,05$ & $0,10 \pm 0,08$ \\
\hline $\mathrm{Cond}$ & $\mu \mathrm{S} / \mathrm{cm}$ & $44,50 \pm 5,66$ & $435,50 \pm 123,08$ & $715,00 \pm 185,23$ \\
\hline Turb & $\mathrm{UNT}$ & $7,50 \pm 6,31$ & $24,55 \pm 252,59$ & $87,00 \pm 355,09$ \\
\hline $\mathrm{SST}$ & $\mathrm{mg} / \mathrm{l}$ & $11,45 \pm 10,57$ & $25,55 \pm 191,34$ & $38,90 \pm 285,88$ \\
\hline $\mathrm{Cl}$ & $\mathrm{mg} / \mathrm{l}$ & $9,86 \pm 2,82$ & $23,15 \pm 2,47$ & $36,71 \pm 3,66$ \\
\hline $\mathrm{NH}_{4}{ }^{-}$ & $\mu \mathrm{g} / \mathrm{l}$ & $2,28 \pm 3,71$ & $11,06 \pm 17,80$ & $21,87 \pm 34,95$ \\
\hline $\mathrm{NO}_{2}^{-}$ & $\mu \mathrm{g} / \mathrm{l}$ & $2,41 \pm 0,87$ & $19,51 \pm 11,32$ & $25,64 \pm 5,02$ \\
\hline $\mathrm{NO}_{3}^{-}$ & $\mu \mathrm{g} / \mathrm{l}$ & $10,64 \pm 8,28$ & $34,43 \pm 22,00$ & $61,52 \pm 16,34$ \\
\hline $\mathrm{PO}_{4}^{-3}$ & $\mu \mathrm{g} / \mathrm{l}$ & $30,71 \pm 24,29$ & $169,49 \pm 29,23$ & $223,62 \pm 59,34$ \\
\hline $\mathrm{DBO}_{5}$ & $\mathrm{mg} / \mathrm{l}$ & $1,75 \pm 1,14$ & $2,78 \pm 0,96$ & $3,50 \pm 1,61$ \\
\hline
\end{tabular}

Fuente: elaboración propia. 
y Decapoda (4 ind.). Análogamente, las familias con más representación en $\mathrm{E}_{1}$ fueron Baetidae (14 ind.), Coenagrionidae (6 ind.), Philopomamidae (5 ind.), Leptohyphidae (4 ind.) y Leptophlebiidae (4 ind.); en $\mathrm{E}_{2}$, las familias más abundantes fueron Culicidae (25 ind.), Hydrophilidae (11 ind.), Coenagrionidae (10 ind.), Psephenidae (5 ind.) e Hydrobiidae (5 ind.). $\mathrm{E}_{3}$ presentó mayor abundancia de organismos en las familias Coenagrionidae (19 ind.), Hydrobiidae (7 ind.), Belastomatidae (5 ind.) y Palaemonidae (4 ind.).

Las asociaciones entre las morfofamilias identificadas en cada estación de monitoreo y las variables fisicoquímicas (figura 4 ) revelan que las familias Baetidae, Isonychiidae, Leptohyphidae, Leptophlebiidae (Ephemeroptera), Calopterygidae, Gomphidae, Platycnemididae (Odononata), Corexidae, Gerridae, Naucoridae, Notonectidae (Hemiptera), Dixidae (Díptera), Heteroceridae (Coleóptera), Hydropsychidae y Philopotamidae
(Tricoptera) se correlacionan positiva y fuertemente con las variables oxígeno disuelto y $\mathrm{pH}$, asociadas a $E_{1}$. Las familias Hidrophilidae, Psephenidae (Coleóptera) Ephemerillidae (Ephemeroptera), Culicidae (Díptera), Planorbidae (Basomatophora), Libellulidae (Odonata) y Tubificidae (Oligochaeta), se asocian más fuertemente con $\mathrm{E}_{2}$. Asimismo, las familias Palaemonidae (Decápoda), Coenagrionidae (Odonata), Chironomidae (Díptera), Hidrobiidae (Sorbeoconcha), Dryopidae (Coleóptera) y Tetragnathidae (Arachnid), se correlacionan fuerte y positivamente con las variables salinidad, turbidez, temperatura, cloruros y los nutrientes $\left(\mathrm{NH}_{4}{ }^{+}\right.$, $\mathrm{NO}_{2}^{-}, \mathrm{NO}_{3}^{-}$y $\mathrm{PO}_{4}^{-3}$ ) en $\mathrm{E}_{3}$.

La estructura numérica de los macroinvertebrados acuáticos colectados en cada estación de monitoreo, a través de los índices de diversidad de Shannon-Weaver y riqueza de Margalef se muestra en la tabla 3. En ella se aprecia que $\mathrm{E}_{1}$ se constituye en un hábitat con una alta diversidad de familias

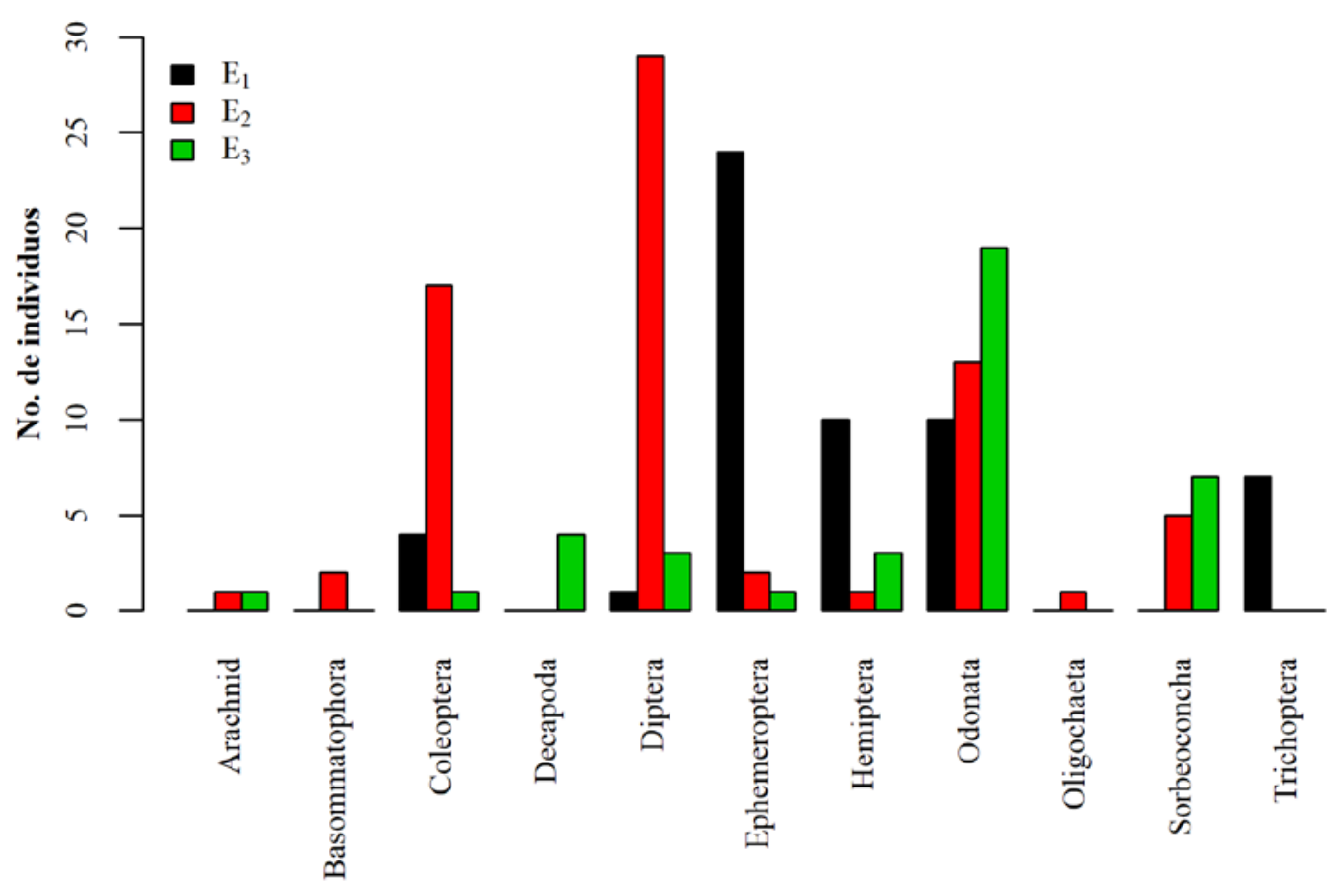

Ordenes

Figura 2. Distribución espacial de los órdenes de macroinvertebrados acuáticos colectados en el área de estudio Fuente: elaboración propia. 


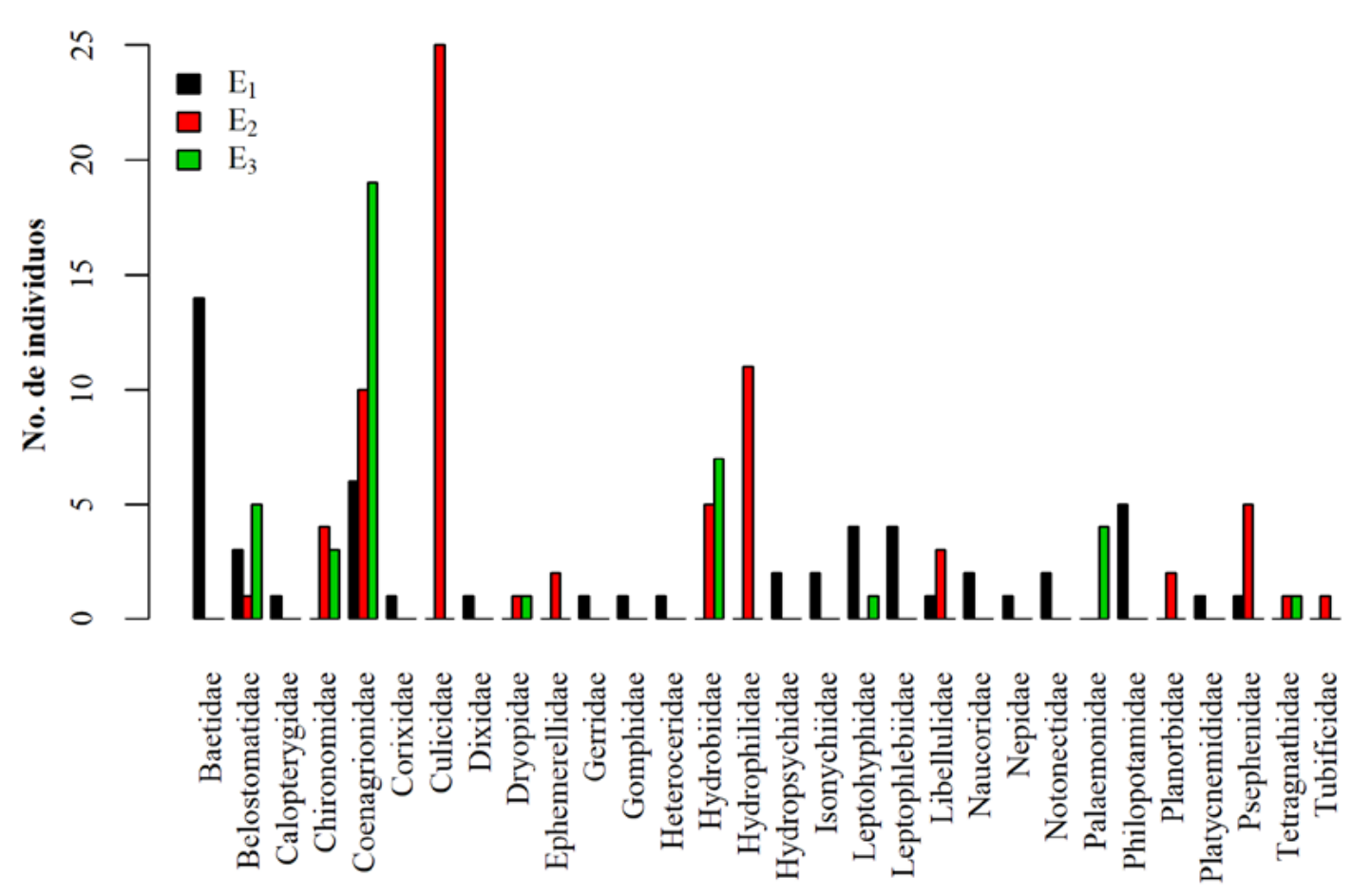

Familias

Figura 3. Distribución espacial de las morfofamilias de macroinvertebrados acuáticos colectados en el área de estudio

Fuente: elaboración propia.

de macroinvertebrados acuáticos $\left(H_{\mathrm{E} 1}^{\prime}=3,75\right.$ bits/ ind), atribuidas a aguas limpias con poca o nula intervención antrópica (Wilhm y Dorris, 1968). Las estaciones $E_{2}$ y $E_{3}$ resultaron ser hábitats con mediana diversidad de familias $\left(H_{\mathrm{E} 2}^{\prime}=2,97\right.$ bits/ind $\mathrm{y}$ $H^{\prime}{ }^{\prime} 3=2,32$ bits/ind), asociadas a ecosistemas con aguas ligeramente contaminadas. La riqueza de familias en la zona de estudio presenta una disminución paulatina conforme el río hace su recorrido de descenso a lo largo de la cuenca, obteniéndose que $\mathrm{E}_{1}$ es la estación con mayor riqueza $\left(I_{\mathrm{E} 1}=\right.$ 4,76), propia de ecosistemas diversos con buena calidad ambiental, mientras que $\mathrm{E}_{2}$ y $\mathrm{E}_{3}$, corresponden con hábitats de mediana riqueza $\left(I_{\mathrm{E} 2}=2,82\right.$ y $\left.I_{\mathrm{E} 3}=1,88\right)$.

El establecimiento de clases de calidad de agua a las estaciones de monitoreo a través del índice biológico BMWP/col y el ASPT se muestra en la tabla 4 , donde se observa que $\mathrm{E}_{1}$ corresponde a la estación con mejor calidad de sus aguas (BWMP/ $\mathrm{col}=130 ; \mathrm{ASPT}=6,50) ; \mathrm{E}_{2}$ y $\mathrm{E}_{3}$ corresponden con aguas de calidad aceptable $(\mathrm{BMWP} / \mathrm{col}=65$; $\mathrm{ASPT}$ $=5,00)$, y dudosa calidad (BMWP/col $=50$; ASPT $=5,25)$, respectivamente. En la figura 5 se muestra el mapa de calidad del agua de la zona de estudio en función del BMWP/col.

Desde el punto de vista biótico, las familias encontradas en $\mathrm{E}_{1}$ son indicadoras de aguas limpias o de buena calidad, caracterizadas por estar bien oxigenadas, con inexistente predominio de procesos de oxidación de materia orgánica (Roldán, 1988; De la Lanza-Espino, Pulido y Pérez, 2000; Hahn-Von Hessberg et al., 2009; Oscoz, Galicia y Miranda, 2009; Rosado, 2009; Flowers y La Rosa, 2010; Gutiérrez y Reinoso-Flórez, 2010; Oliveira y Callisto, 2010; Ramírez, 2010; Forero, 2013; Gil et 


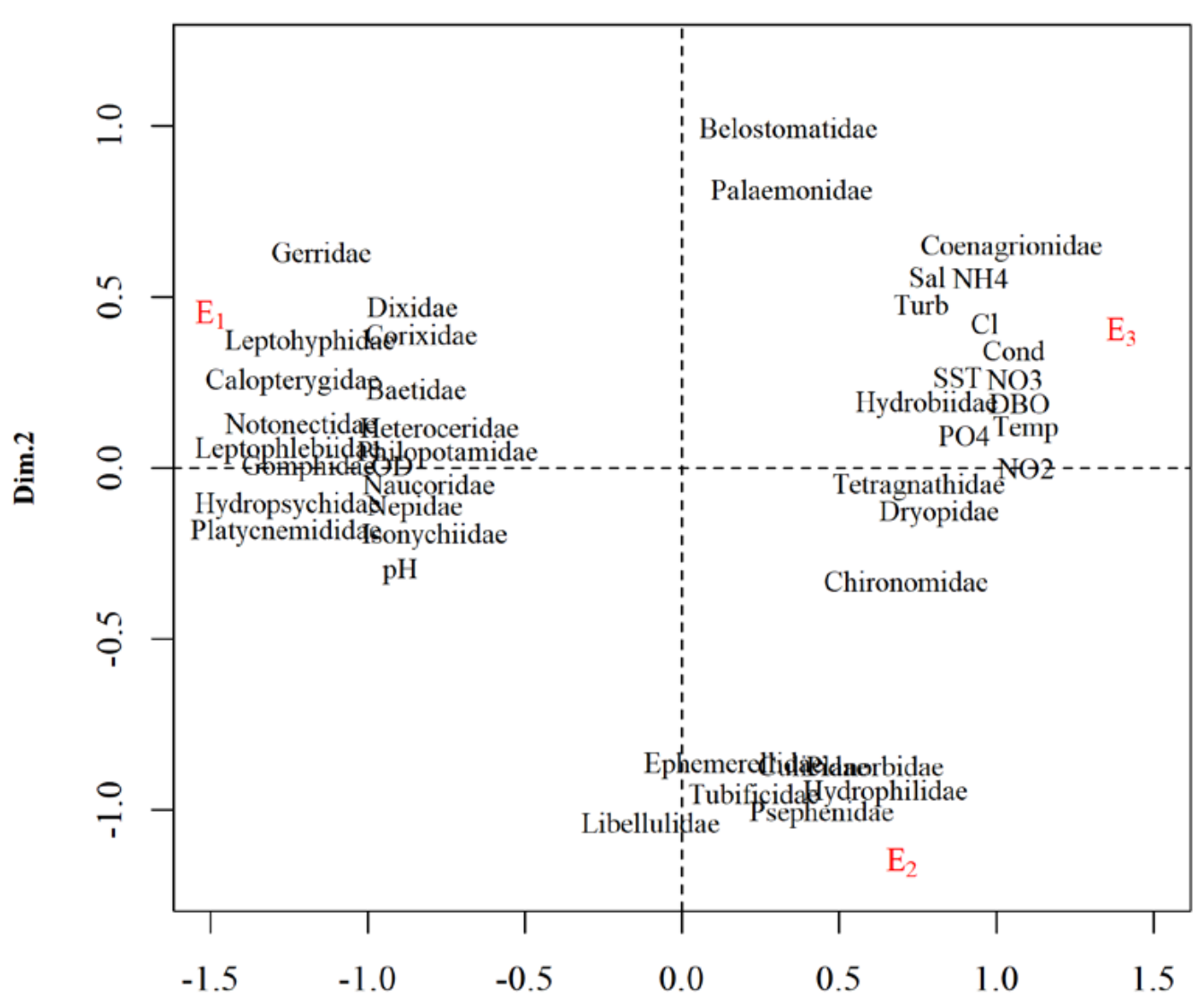

Dim.1

Figura 4. Asociaciones entre morfofamilias identificadas y las variables fisicoquímicas

Fuente: elaboración propia.

Tabla 3. Estructura numérica de los macroinvertebrados acuáticos colectados

\begin{tabular}{ccc}
\hline Estación & $\begin{array}{c}\text { Índice de diversidad de Shannon- } \\
\text { Weaver }\left(\mathbf{H}^{\prime}\right)(\text { bits/ind })\end{array}$ & Índice de riqueza de Margalef $(\boldsymbol{I})$ \\
\hline $\mathrm{E}_{1}$ & 3,75 & 4,76 \\
\hline $\mathrm{E}_{2}$ & 2,97 & 2,82 \\
\hline $\mathrm{E}_{3}$ & 2,32 & 1,88 \\
\hline
\end{tabular}

Fuente: elaboración propia.

Tabla 4. Calidad del agua de la zona de estudio según el BMWP/col

\begin{tabular}{cccl}
\hline Estación & BMWP/col & ASPT & Calidad \\
\hline $\mathrm{E}_{1}$ & 130 & 6,50 & Aguas de clase I, de buena calidad o no contaminadas. \\
\hline $\mathrm{E}_{2}$ & 65 & 5,00 & Aguas clase III, de calidad aceptable o ligeramente contaminadas. \\
\hline $\mathrm{E}_{3}$ & 50 & 5,25 & Aguas clase IV, de calidad dudosa o moderadamente contaminadas. \\
\hline
\end{tabular}

Fuente: elaboración propia. 
al., 2013; González-Soriano y Novelo-Gutiérrez, 2014; Jiménez, 2014). En $\mathrm{E}_{2}$, las familias identificadas son indicadoras de estrés en el ecosistema, tolerantes a aguas menos oxigenadas, de bajo caudal y con aportes de materia orgánica (Roldán, 1988; Oscoz, Galicia y Miranda, 2009; Rosado, 2009); no obstante, a la existencia de familias representantes de los órdenes Ephemeroptera y Coleóptera, estas consisten en familias relativamente tolerantes a niveles de estrés por materia orgánica (Oscoz, Galicia y Miranda, 2009). Las familias encontradas en E3 son en su mayoría indicativas de aguas con moderada contaminación de origen de orgánico (Oscoz, Galicia y Miranda, 2009; Rosado, 2009) y/o aguas oligomesotróficas (Rosado, 2009). Se destaca la presencia de representantes de la familia Palaemonidae, considerada como indicadora de buena calidad (Roldán-Pérez, 2003). Sin embrago, De la Lanza-Espino, Pulido y Pérez
(2000) consideran a esta familia como indicadoras de bajas concentraciones de oxígeno, altas temperaturas y falta de vegetación riparia, condiciones imperantes en esta estación de monitoreo.

Los valores de diversidad de macroinvertebrados acuáticos en $\mathrm{E}_{1}$ son semejantes a los reportados para ecosistemas lóticos con poca intervención antrópica y mayor variedad de microhábitats (Arango et al., 2008; Chalá et al., 2003). Asimismo, la clasificación de calidad del agua en función al índice $\mathrm{BMWP} / \mathrm{col}$ es similar a los valores establecidos en una quebrada del oriente antioqueño con aguas muy limpias o de buena calidad que, junto a una gran variedad de biotopos, la disponibilidad de alimento y las características del sustrato (similares a $\mathrm{E}_{1}$ ), permiten una gran abundancia y distribución de las comunidades de macroinvertebrados acuáticos, de allí su alta diversidad y riqueza (Moreno, 2008).

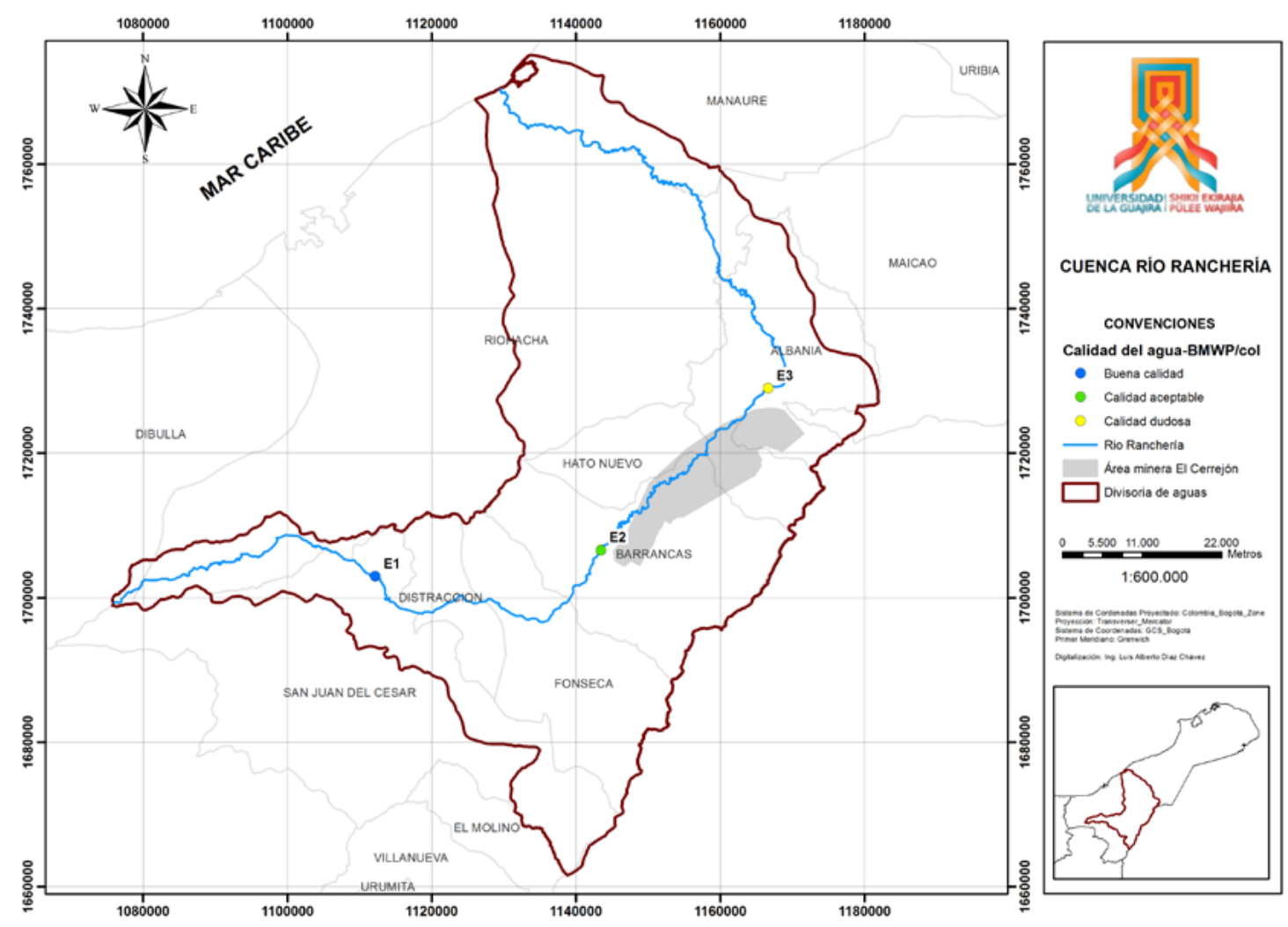

Figura 5. Mapa de calidad de agua de la zona de estudio según BMWP/col

Fuente: elaboración propia. 
En $E_{2}$ y $E_{3}$ la disminución paulatina de la diversidad y riqueza de la fauna de macroinvertebrados acuáticos se explica por la perturbación del ecosistema debido a factores contaminantes como el urbanismo y sus actividades conexas, donde el aumento de los sólidos y la turbidez (como ocurre en río Ranchería) son variables limitantes para el establecimiento de la fauna de macroinvertebrados, dado que se impide el paso de rayos solares al medio, se afecta y tapona el sistema de intercambio gaseoso en los animales acuáticos y destruyen sus hábitats naturales; afectando directamente a las comunidades vegetales que sirven de alimento y refugio para los insectos (Garcia-Alzate, Román-Valencia, González, \& Barrero, 2010).

En consecuencia, se refleja el decaimiento de la calidad ambiental del ecosistema a través del BWMP/col. No obstante, la clasificación de la calidad del agua en estas estaciones está ligeramente afectada en comparación a la zona baja de la cuenca, donde los valores de bioindicación a través del mismo índice, adaptado a las condiciones del ecosistema estuarino del río Ranchería, clasifica sus aguas como de clase III, de calidad crítica o aguas muy contaminadas (Castro et al., 2013).

\section{CONCLUSIONES}

Los resultados obtenidos son concluyentes en que la valoración de la calidad del ecosistema, a través de los macroinvertebrados acuáticos y las variables fisicoquímicas analizadas, revela un deterioro progresivo de la salud ambiental del río Ranchería, conforme se avanza en el recorrido del río desde aguas arriba hasta la zona más baja. La estación $\mathrm{E}_{1}$, tomada como blanco, se clasificó como aguas limpias o de buena calidad según la aplicación del índice BMWP/col, con predominio de morfofamilias de macroinvertebrados asociadas a aguas limpias con buena oxigenación. Las estaciones $E_{2}$ y $E_{3}$ se clasificaron como aguas de calidad aceptable y dudosa, respectivamente. Lo anterior explica la pérdida de diversidad y riqueza de las familias de macroinvertebrados, con dominio de comunidades de macroinvertebrados asociados a las condiciones de degradación ambiental que sufre el medio.

\section{FINANCIAMIENTO}

Este artículo es producto del proyecto "Salud ambiental del río Ranchería a través de macroinvertebrados acuáticos en el área de influencia del complejo carbonífero El Cerrejón", investigación avalada y financiada por la Universidad de La Guajira.

\section{AGRADECIMIENTOS}

Los autores agradecen a la Universidad de La Guajira, por el apoyo logístico y financiero, y al Grupo de Investigación Pichihuel, por el acompañamiento en las actividades de monitoreo.

\section{REFERENCIAS}

Alba-Tercedor, J. y Sánchez-Ortega, A. (1988). Un método rápido y simple para evaluar la calidad biológica de las aguas corrientes basado en el de Hellawell (1978). Limnética, 4(5), 1-56.

Álvarez, A. L. (2005). Metodología para la evaluación de los macroinvertebrados acuáticos como indicadores de los recursos hidrobiológicos. Bogotá: Instituto Alexander Von Humboldt.

Álvarez, L. F. y Roldán, G. (1983). Estudio del orden Hemiptera (Heteroptera) en el departamento de Antioquia en diferentes pisos altitudinales. Actualidades Biológicas, 12(44), 31-46.

American Public Health Association (APHA) et al. (2005). Standard methods for the examination of water and wastewater. Washington, D. C., EE. UU.

Aquatic National (2001). A pictorial guide to the orders of aquatic invertebrates. Logan, Utah: Department of Fisheries and Wildlife, Utah State University.

Arango, M. C. y Roldán, G. (1983). Odonatos inmaduros del departamento de Antioquia en diferentes pisos altitudinales. Actualidades Biológicas, 12(46), 91-105. 
Arango, M. C., Álvarez, L. F., Arango, G. A., Torres, O. E. y Monsalve, A. de J. (2008). Calidad del agua de las quebradas la Cristalina y la Risaralda, San Luis, Antioquia. Revista EIA, (9), 121-141.

Argumedo, C. D., Gómez, J. y Negrete, J. M. (2017). Metales en sedimento del río Ranchería, La Guajira. Bistua, Revista de la Facultad de Ciencias Básicas, 15(2), 64-68.

Arteta-Bonivento, R. (2009). Crabs in the rio Rancheria Delta, Riohacha (Colombia) (Crustacea: Decapoda: Brachyura). Boletín Científico. Centro de Museos. Museo de Historia Natural, 13(1), 140-152.

Bedoya, O. I. y Roldán, P. G. (1984). Estudio de los dípteros acuáticos en diferentes pisos altitudinales del departamento de Antioquia. Revista de la Asociación Colombiana de Ciencias Biológicas, 2(2), 113-134.

Bolívar, G. E. M., Pitre, I. A. J. y Correa, D. A. (2017). Cuantificación de coliformes totales en estuario del río Ranchería. Revista Biotecnología, UniCacuca, 15(2), 23-29. 10.18684/bsaa(15).590

Bonivento, Z. (2010). Evaluación de la calidad del agua en el marco del diagnóstico del estado del río Ranchería en el año 2010 (Tesis de pregrado). Universidad de La Guajira, Riohacha, Colombia.

Bouchard, R. W., Ferrington, L. C. y Karius, M. L. (2004). Guide to aquatic invertebrates of the Upper Midwest. Water Resources Center, University of Minnesota, St. Paul, MN. 208 p.

Castañé, P. M., Loez, C. R., Olgin, H. F., Puig, A., Rovedatti, M. G., Topalián, M. L. y Salibián, A. (1998). Caracterización y variación espacial de parámetros fisicoquímicos y del plancton en un río urbano contaminado (río Reconquista, Argentina). Revista Internacional de Contaminación Ambiental, 14(2), 69-77.

Castro, F., Rosado, J., Deluque, M., Jiménez, J. y Madrugo, E. (2005). Estudio limnológico del ecosistema estuarino "El Riito", transecto del río Ranchería, Riohacha - La Guajira. Riohacha: Universidad de La Guajira.

Castro, F., Rosado, J., Ríos, H., De Armas, N. y CasteIlanos, K. (2013). Evaluación de la calidad ecológica del ecosistema Riito, transecto del tío Ranchería,
Riohacha, La Guajira. Riohacha: Universidad de La Guajira.

Corporación Autónoma Regional de La Guajira (Corpoguajira) (2011). Plan de Ordenamiento de la cuenca del río Ranchería. Riohacha.

Corporación Autónoma Regional de La Guajira (Corpoguajira) y Fundación Pro-Sierra Nevada de Santa Marta. (2012). Plan de ordenamiento del recurso hídrico y reglamentación de corrientes y vertimientos de las cuencas de los ríos Ranchería, Carraipía y Tomarrazón-Camarones, en el departamento de La Guajira. Riohacha.

Correa, M., Machado, T. y Roldán, G. (1981). Taxonomía y ecología del orden Trichoptera en el departamento de Antioquia en diferentes pisos altitudinales. Actualidades Biológicas, 10(36), 35-48.

Chalá, W. P., Garca, D. M., Mosquera, M. L., Caicedo, K. P., Palacios, J. A., Castro, A. A. y Guerrero, J. E. (2003). Diversidad de macroinvertebrados y evaluación de la calidad del agua de la quebrada La Bendición, municipio de Quibdó (Chocó-Colombia). Acta Biológica Colombiana, 8(2), 23-30.

De la Lanza-Espino, G., Pulido, S. H. y Pérez, J. L. C. (2000). Organismos indicadores de la calidad del agua y de la contaminación (bioindicadores). Madrid: Plaza y Valdés.

Encalada, A. (2010). Funciones ecosistémicas y diversidad de los ríos: Reflexiones sobre el concepto de caudal ecológico y su aplicación en el Ecuador. Polemika, 2(5), 40-47.

Fernández, H. y Domínguez, E. (2001). Guía para la determinación de los artrópodos bentónicos Sudamericanos. Universidad Nacional de Tucumán. Facultad de Ciencias Naturales e Instituto M. Lillo, Tucumán. 282 p.

Flowers, R. W. y La Rosa, C. (2010). Capítulo 4: Ephemeroptera. Revista de Biología Tropical, 58, 63-93.

Forero, A. M. (2013). Estudio de la familia Baetidae (Ephemeroptera: Insecta) en una cuenca con influencia de la urbanización y agricultura: río Alvarado-Tolima. Revista de la Asociación Colombiana de Ciencias Biológicas, 1(25), 12-21. 
Fuentes, G. A., Verbel, J. O., Villegas, J. C. V. y Campos, D. A. (2019). Si el río suena, piedras lleva: Sobre los derechos al agua y a un ambiente sano en la zona minera de La Guajira. Indepaz. 164 p. Recuperado de http://www.indepaz.org.co/wp-content/ uploads/2019/02/Si-el-rio-suena-piedras-Ileva-Indepaz-2019.pdf

Garbagnati, M. A., González, P. S., Antón, R. I. y MaIlea, M. A. (2005). Características fisicoquímicas, capacidad buffer y establecimiento de la línea base ambiental del río Grande, San Luis, Argentina. Ecología Austral, 15, 59-71.

García-Alzate, C. A., Román-Valencia, C., González, M. I. y Barrero, A. M. (2010). Composición y variación temporal de la comunidad de insectos acuáticos (Insecta) en la quebrada Sardineros, afluente Río Verde, Alto Cauca, Colombia. Revista de Investigaciones de La Universidad de Quindío, 21, 21-28.

Giacometti, J. C. (2019). Macroinvertebrados acuáticos y su importancia como bioindicadores de calidad del agua en el río Alambi. Boletín Técnico, Serie Zoológica, 6(2), 17-32.

Gil, Z. N., Bustillo, A. E., Gómez, N., García, P. A. y Zuluaga, Y. M. (2013). Las libélulas y su rol en el ecosistema de la zona cafetera. Centro nacional de investigaciones del café (CENICAFE), Avances Técnicos 357, 8 p.

González, L. y Lozano, L. (2004). Bioindicadores como herramienta de evaluación de la calidad ambiental en la parte alta de la microcuenca las delicias. Umbral Científico, (5), 73-82.

González-Soriano, E. y Novelo-Gutiérrez, R. (2014). Biodiversidad de Odonata en México. Revista Mexicana de Biodiversidad, 85, 243-251. 10.7550/ rmb.34716

Guerrero-Bolaño, F., Manjarrés-Hernández, A. y Núñez-Padilla, N. (2003). Los macroinvertebrados bentónicos de Pozo Azul (cuenca del río Gaira, Colombia) y su relación con la calidad del agua. Acta Biológica Colombiana, 8(2), 43-55.

Guinard, J. D. C., Ríos, T. y Vega, J. A. B. (2013). Diversidad y abundancia de macroinvertebrados acuáticos y calidad del agua de las cuencas alta y baja del río Gariché, provincia de Chiriqui, Panamá. Gestión y Ambiente, 16(2), 61-70.

Gutiérrez, C. y Reinoso-Flórez, G. (2010). Géneros de ninfas del orden Ephemeroptera (Insecta) del departamento del Tolima, Colombia: listado preliminar. Biota Colombiana, 11(1 y 2), 23-32.

Gutiérrez, J. D., Riss, W. y Ospina, R. (2004). Bioindicación de la calidad del agua con macroinvertebrados acuáticos en la sabana de Bogotá, utilizando redes neuronales artificiales. Caldasia, 26(1), 151-160.

Hahn-Von Hessberg, C., Toro, D., Grajales-Quintero, A., Duque-Quintero, G. y Serna-Uribe, L. (2009). Determinación de la calidad del agua mediante indicadores biológicos y fisicoquímicos, en la estación piscícola, Universidad de Caldas, Municipio de Palestina, Colombia. Boletín Científico Centro de $\mathrm{Mu}$ seos. Museo de Historia Natural, 13(2), 89-105.

Jiménez, D. M. (2014). Aspectos ecológicos de la familia leptohyphidae (insecta: ephemeroptera) de la cuenca del río Alvarado (Tolima, Colombia) (Tesis de pregrado). Universidad del Tolima, Ibagué, Colombia.

López, C. N., Lazo, D. G., González, G. G., Brandimarte, A. L., Riveaux, S. M. y Cordero, Y. M. (2005). Una metodología rápida y de fácil aplicación para la evaluación de la calidad del agua utilizando el índice BMWP-Cub para ríos cubanos. Tecnura, 9(17), 65-76.

Machado, T. y Rincón, J. (1989). Distribución ecológica e identificación de los coleópteros acuáticos en diferentes pisos altitudinales del departamento de Antioquia (Tesis de pregrado). Universidad de Antioquia, Medellín, Colombia.

Madera, L. C., Angulo, L. C., Díaz, L. C. y Rojano, R. (2016). Evaluación de la calidad del agua en algunos puntos afluentes del río Cesar (Colombia) utilizando macroinvertebrados acuáticos como bioindicadores de contaminación. Información Tecnológica, 27(4), 103-110. 10.4067/ S0718-07642016000400011

McCafferty, W. P. (2014). Aquatic entomology: the fishermen's and ecologists' illustrated guide to insects and their relatives. Burlington, Massachusetts: Jones \& Bartlett Learning. 
Medianero, E. y Samaniego, M. (2004). Comunidad de insectos acuáticos asociados a condiciones de contaminación en el río Curundú, Panamá. Folia Entomológica Mexicana, 43(3), 279-294.

Melo, M. C. y Montemayor, S. I. (2014). Rhopalidae. Biodiversidad de Artrópodos Argentinos, 3, 451-456.

Molina, C. I., Fossati, O. y Marín, R. (2006). Ensayo de un método para el estudio de macroinvertebrados acuáticos en un río contaminado de la cuidad de la Paz-Bolivia. Ciencia Abierta Internacional, 29, 1-14.

Molina-Bolívar, G. y Jiménez-Pitre, I. (2017). Análisis de la contaminación por coliformes termotolerantes en el estuario del río Ranchería, La Guajira (Colombia). Boletín Científico. Centro de Museos. Museo de Historia Natural, 21(2), 41-50. 10.18684/ bsaa(15).590

Moreno, Y. M. (2008). Caracterización de la biodiversidad acuática y de la calidad de las aguas de la quebrada Los Andes, El Carmen de Viboral, Antioquia. Revista Institucional Universidad Tecnológica del Chocó, 27(1), 85-91.

Muñoz Quesada, F. J. (2004). El orden Trichoptera (Insecta) en Colombia, II: inmaduros y adultos, consideraciones generales. En F. Fernández, M. G. Andrade, y G. Amat (Eds.) Insectos de Colombia Vol III.(pp. 319-349). Universidad Nacional de Colombia-Instituto Humboldt. Bogotá, D.C.

Murillo-Montoya, S. A., Mendoza-Mora, A., Restrepo-Bastidas, E. S. y Rodríguez, M. Á. (2018). Utilización de macroinvertebrados acuáticos como herramienta para determinar la calidad del agua en la quebrada Santo Tomás, municipio de Pensilvania, Colombia. Revista de la Academia Colombiana de Ciencias Exactas, Físicas y Naturales, 42(164), 212-220. 10.18257/raccefyn.655

Oliveira, A. y Callisto, M. (2010). Benthic macroinvertebrates as bioindicators of water quality in an Atlantic forest fragment. Iheringia. Série Zoologia, 100(4), 291-300. 10.1590/S0073-47212010000400003

Ortega, M. et al. (1988). Características fisicoquímicas de las aguas superficiales de la Rambla del Moro después de una riada (cuenca del río Segura, SE de
España). Limnética, 4, 19-26. Recuperado de http:// www.limnetica.net/Limnetica/Limne04/L04u019 Fisicoquimica_aguas_rambla_Moro.pdf

Ortiz, L. L. (2005). La bioindicación de la calidad del agua: importancia de los macroinvertebrados en la cuenca alta del río Juan Amarillo, Cerros Orientales de Bogotá. Umbral Cientifico, (7), 5-11.

Oscoz, J., Galicia, D. y Miranda, R. (2009). Macroinvertebrados de la cuenca del Ebro: descripción de taxones y guía de identificación. Pamplona, España: Departamento de Zoología y Ecología, Universidad de Navarra.

Pérez, G. R. (1999). Los macroinvertebrados y su valor como indicadores de la calidad del agua. Academia Colombiana de Ciencia, 23(88), 375-387.

Pérez, J. I., Nardini, A. G. y Galindo, A. A. (2018). Análisis comparativo de índices de calidad del agua aplicados al río Ranchería, La Guajira-Colombia. Información Tecnológica, 29(3), 47-58. 10.4067/ S0718-07642018000300047

Posada, G. et al. (2000). Caracterización fisicoquímica y biológica de la calidad de aguas de la cuenca de la quebrada Piedras Blancas, Antioquia, Colombia. Revista de Biología Tropical, 48(1), 59-70.

Ramírez, A. (2010). Capítulo 5: Odonata. Revista de Biología Tropical, 58, 97-136.

Rangel, M. (2013). Evaluación de la calidad del agua utilizando índices pertinentes a los usos del río Ranchería, departamento de La Guajira - Colombia (Tesis de pregrado). Universidad de La Guajira, Riohacha, Colombia.

Riss, W., Ospina, R. y Gutiérrez, J. D. (2002). Establecimiento de valores de bioindicación para macroinvertebrados acuáticos de la sabana de Bogotá. Caldasia, 24(1), 135-156.

Roldán, G. (1985). Contribución al conocimiento de las ninfas de los efemerópteros (clase; insecta, orden: Ephemeroptera) en el departamento de Antioquia, Colombia. Actualidades Biológicas, 14(51), 3-13.

Roldán, G. (1988). Guía para el estudio de los macroinvertebrados acuáticos del Departamento de Antioquia. Medellín: Universidad de Antioquia, Fondo FEN. 
Roldán-Pérez, G. (2003). Bioindicación de la calidad del agua en Colombia: propuesta para el uso del método BMWP Col. Medellín: Universidad de Antioquia.

Roldán-Pérez, G. (2016). Los macroinvertebrados como bioindicadores de la calidad del agua: cuatro décadas de desarrollo en Colombia y Latinoamérica. Revista de la Academia Colombiana de Ciencias Exactas, Físicas y Naturales, 40(155), 254-274. 10.18257/raccefyn.335

Rosado, J. (2009). Laguna Salá: su biología y ambiente. 2a. ed. Riohacha, Colombia: Editorial Gente Nueva. Sánchez-Vélez, A. y García-Núñez, R. M. (1999). Biomonitoreo de ríos en la gestión de cuencas: una aproximación introductoria. IX Congreso Nacional de Irrigación, Memorias de Simposio 4 sobre Manejo Integral de Cuencas Hidrográficas, 63-71.

Shannon, C. E. (1948). A mathematical theory of communication. Bell System Technical
Journal,27(3),379-423.10.1002/j.1538-7305.1948. tb01338.x

Toro, M. et al. (2002). Calidad de las aguas de los ríos mediterráneos del proyecto Guadalmed. Características fisicoquímicas. Limnética, 21(3-4), 63-75.

Vivas, S., Casas, J., Pardo, I., Robles, S., Bonada, N., Mellado, A. et al. (2002). Aproximación multivariante en la exploración de la tolerancia ambiental de las familias de macroinvertebrados de los ríos mediterráneos del proyecto Guadalmed. Limnética, 21(3-4), 149-173.

Wilhm, J. \& Dorris, T. (1968). Biological parameter of wáter quality. Bioscence, 18, $477-481$. $10.2307 / 1294272$

Zúñiga de Cardoso, M. del C. (1985). Estudio de la ecología del río Cali con énfasis en su fauna bentónica como indicador biológico de calidad. Revista Ainsa, 5(1), 63-85.

\section{(c) (1) ()}

